\title{
INITIAL PERFORMANCE OF THE IUCF COOLER INJECTOR SYNCHROTRON ${ }^{*}$
}

\author{
D.L Friesel, ${ }^{\#}$ M. Ball, B. Hamilton, Wm. Manwaring, and T. Sloan \\ IUCF, Bloomington, IN
}

\section{Abstract}

Construction of a compact $2.4 \mathrm{Tm}$ Cooler Injector synchrotron (CIS) to injection intense polarised proton and deuteron beams into the IUCF $3.6 \mathrm{Tm}$ electron cooled storage ring (Cooler) is complete. Beam commissioning began in 1997 with unpolarized $\mathrm{H}^{-}$strip injection and progressed to the extraction of $210 \mathrm{MeV}$ protons from CIS and the injection of $10^{10}$ protons at 200 $\mathrm{MeV}$ into the Cooler for experiment. With a circumference of $17.36 \mathrm{~m}$, CIS is possibly the smallest and least expensive example of this accelerator type for protons, and may serve as a practical proton source for medical and other applications. This contribution discusses our design choices, the resulting technical and fabrication challenges, and their solutions. Emphasis is given to the CIS ring beam performance from7 $\mathrm{MeV} \mathrm{H}^{-}$ injection through Cooler $200 \mathrm{MeV}$ proton injection and accumulation.

\section{INTRODUCTION}

A compact synchrotron to replace the Indiana k220 cyclotron as an injector of polarised protons and deuterons into the Cooler [1] was jointly funded by Indiana University $(\$ 1.5 \mathrm{M})$ and the NSF Academic Research Infrastructure Program $(\$ 2.0 \mathrm{M})$ in August of 1994. The primary goal was to inject over $10^{10}$ polarised particles/pulse into the Cooler to improve experimental luminosity for medium energy cooled beams on thin internal targets. Initial CIS design parameters were influenced by the relatively low intensities available from pulsed polarised ion sources $(\approx 1 \mathrm{~mA}$ peak) and by the desire to avoid an intrinsic depolarising resonance in the Cooler for $186 \mathrm{MeV}$ protons. A secondary goal was to simplify the Cooler injection process for polarised beams to reduce machine overhead and increase experimental duty factor. Finally, only limited space was available in the existing facility to house a new injector accelerator. These requirements dictated negative ion strip injection into a small synchrotron with single turn fast extraction and bucket-to-bucket kick injection into the Cooler. The injection and extraction energies ( $7 \mathrm{MeV} \mathrm{H}$ and $200 \mathrm{MeV} \mathrm{P}$ ) were the minimum required to meet the intensity and resonance goals. The

"Work supported by the National Science Foundation. and Indiana University

${ }^{\#}$ Email: Friesel@IUCF.indiana.edu
Table I: CIS Booster Design Parameters

\begin{tabular}{|l|l|l|}
\hline Polarised Source & $25 \mathrm{keV} \mathrm{H}^{-}$ & $25 \mathrm{keV} \mathrm{d}^{-}$ \\
\hline Linac Pre-accelerator & $7 \mathrm{MeV} \mathrm{H}$ & $4 \mathrm{MeV} \mathrm{d}^{-}$ \\
\hline Strip Injection Foil & $4.5 \mu \mathrm{gm} / \mathrm{cm}^{2} \mathrm{C}$ & $3.0 \mu \mathrm{gm} / \mathrm{c}^{2} \mathrm{C}$ \\
\hline Fast Extraction & $220 \mathrm{MeV} \mathrm{P}$ & $105 \mathrm{MeV} \mathrm{d}$ \\
\hline Extracted Intensity & $\geq 10^{10} \mathrm{p} / \mathrm{pulse}$ & $\geq 5 \times 10^{9} \mathrm{~d} / \mathrm{p}$ \\
\hline Rep. Rate & 1 to $5 \mathrm{~Hz}$ & 1 to $5 \mathrm{~Hz}$ \\
\hline
\end{tabular}

resulting design parameters for CIS are listed in Table I. With a budget of $\$ 3.5 \mathrm{M}$, only the Linac, RF cavity, and ring magnetic, diagnostic and extraction system were designed and fabricated specifically for CIS. The majority of the remaining systems (temporary $\mathrm{H}^{-}$source, injection and extraction beam lines, ring vacuum system, etc) were assembled from surplus equipment available either in-house or from other laboratories (FNAL, AGNL, etc).

\section{CIS CONFIGURATION}

A schematic of the present IUCF nuclear research accelerator facilities, consisting of the new CIS booster synchrotron and the electron cooler storage ring, is shown in Fig. 1. A $25 \mathrm{keV} \mathrm{H}$ beam is matched to the acceptance of a commercial $7 \mathrm{MeV} \mathrm{H}$ linac preaccelerator consisting of a $3 \mathrm{MeV}$ RFQ coupled to a 4 $\mathrm{MeV}$ DTL [2,3]. Both operate at $425 \mathrm{MHz}$. The $7 \mathrm{MeV}$ $\mathrm{H}^{-}$beam is strip injected into the synchrotron, protons are accumulated, RF captured, accelerated to $210 \mathrm{MeV}$ and single turn fast extracted for bucket-to-bucket transfer

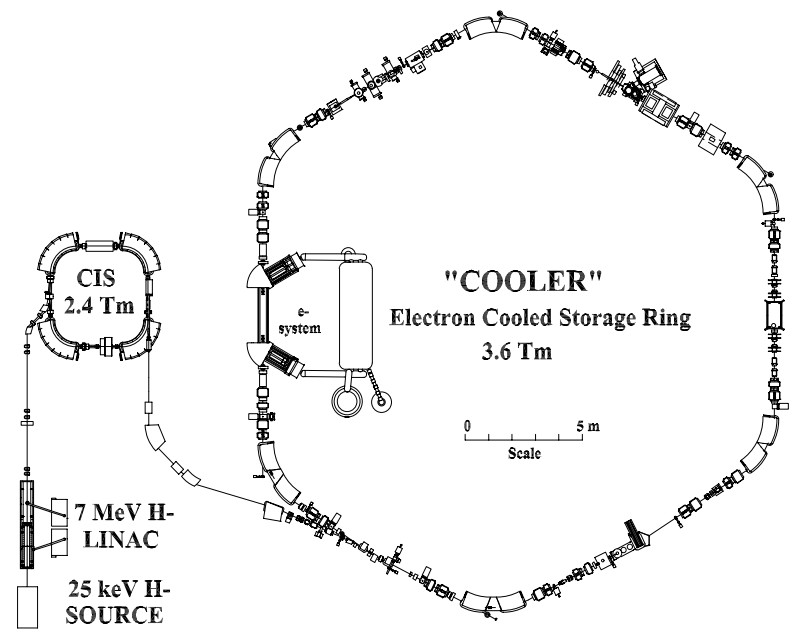

Figure1: Present IUCF Accelerator Research Facility 
into the Cooler ring for accumulation, electron cooling and further acceleration.

\subsection{Polarised Ion Source}

A high intensity polarised negative ion source $(~<1 \mathrm{~mA}$ $\mathrm{H}$, d peak) with a colliding beam ionizer (CIPIOS) [4] was fabricated simultaneously with CIS, and was recently (3/99) installed in the CIS vault. Injection of polarised $\mathrm{H}$ beams from this new source, which is also capable of delivering polarised $\mathrm{d}^{-}$ions, is scheduled to begin in June 1999. Deuteron beam pre-acceleration for CIS injection is done by replacing the $3 \mathrm{MeV} H$ RFQ vanes with another set designed to accelerate $q / A=1 / 2$ ions to $4 \mathrm{MeV}$. The RFQ vacuum vessel and $\mathrm{H}^{-}$vane assembly were fabricated with this replacement capability built in. The $\mathrm{q} / \mathrm{A}=1 / 2$ vane assembly is now being fabricated by AccSys Technology, Inc [5] with delivery scheduled for June 1999. Following initial installation and alignment of the new vanes (late 1999), switching from $\mathrm{H}^{-}$to $\mathrm{d}^{-}$operation will likely take 2 days. The CIS/Cooler $\mathrm{H}$ beam commissioning studies reported here were conducted using a surplus $0.5 \mathrm{~mA}$ (peak) unpolarized Duoplasotron source [6].

\subsection{The CIS Lattice Design}

A schematic of the CIS ring is shown in Fig. 2 and the lattice parameters are listed in Table II. CIS is a weak focusing synchrotron with optical properties determined by the ring dipole-straight length ratio and the dipole $12^{\circ}$ edge angles. A quadrupole was installed in each straight to correct for dipole edge angle fabrication errors and to study ring performance at transition by making large tune changes. The lattice, linac pre-accelerator and major accelerator system hardware designs were previously reported $[7,8,9]$. Each of the four $2.34 \mathrm{~m}$ long straight sections are filled with apparatus required for $\mathrm{H}^{-}$

Table II: Lattice Parameters for the CIS Ring

\begin{tabular}{|c|c|}
\hline Circumference & $17.364 \mathrm{~m}$ \\
\hline Betatron Tunes; Qx, Qy & $1.478,0.72$ \\
\hline Dipole Length & $2.0 \mathrm{~m}$ \\
\hline Dipole Edge Angle & $12^{\circ}$ \\
\hline Dipole Bend radius & $1.273 \mathrm{~m}$ \\
\hline $\begin{array}{ll}\beta x: & \begin{array}{l}\text { Maximum } \\
\text { Minimum }\end{array}\end{array}$ & $\begin{array}{l}4.373 \mathrm{~m} \\
0.996 \mathrm{~m}\end{array}$ \\
\hline $\begin{array}{l}\text { Maximum } \\
\text { Minimum }\end{array}$ & $\begin{array}{l}3.786 \mathrm{~m} \\
3.380 \mathrm{~m}\end{array}$ \\
\hline $\begin{array}{ll}\text { Dispersion: } & \begin{array}{l}\text { Maximum } \\
\text { Minimum }\end{array} \\
\end{array}$ & $\begin{array}{l}1.759 \mathrm{~m} \\
1.617 \mathrm{~m} \\
\end{array}$ \\
\hline $\begin{array}{ll}\text { Chromaticity: } & \mathrm{Cx} \\
& \mathrm{Cz} \\
\end{array}$ & $\begin{array}{l}-0.529 \\
-0.156 \\
\end{array}$ \\
\hline Transition Energy & $256 \mathrm{MeV}$ \\
\hline
\end{tabular}

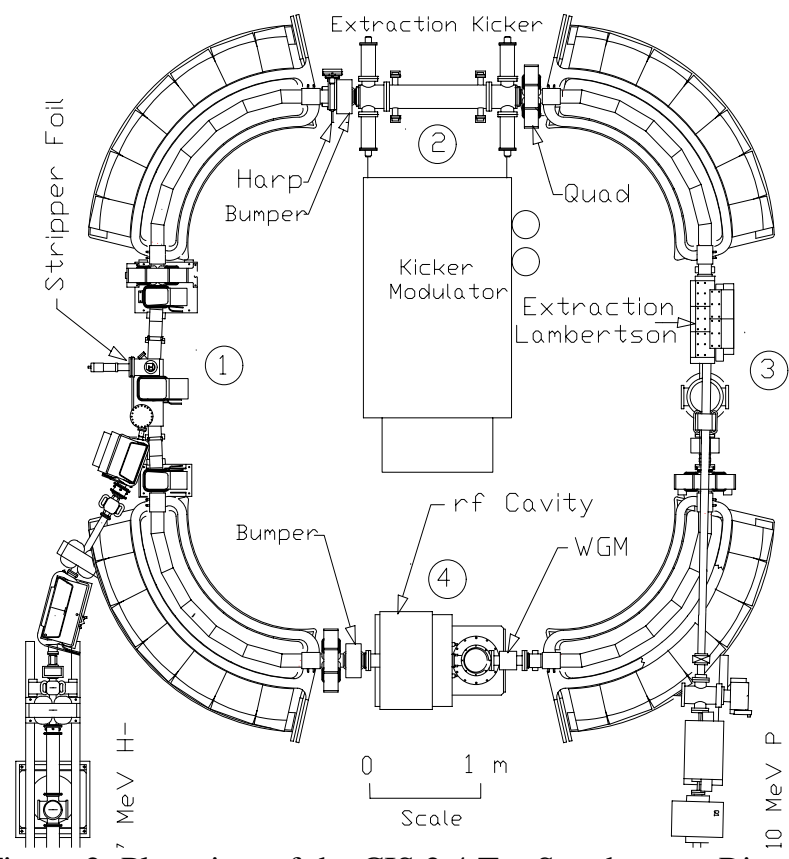

Figure 2: Plan view of the CIS 2.4 Tm Synchrotron Ring.

strip injection (1), single-turn kick extraction ( $2 \& 3)$ and RF acceleration (4). Five vertical steering elements distributed around the ring and four main dipole trim coils are used for beam centering. Beam diagnostics consist of an x/y BPM pair placed at the entrance and exit of each dipole, a high bandwidth wall gap monitor, a ping tune kicker and a removable wire Harp to view circulating beam during injection [10].

\subsection{Fabrication Challenges \& Innovations}

The energy and small circumference $\left(1 / 5^{\text {th }}\right.$ Cooler $)$ of CIS precipitated significant design and engineering challenges for the ring main dipoles and the fast extraction system. The ring design requires $90^{\circ}$ laminated dipoles with a $1.273 \mathrm{~m}$ radius (37 $\mathrm{cm}$ sagitta) to ramp from 0.3 to $1.68 \mathrm{~T}$ at up to $5 \mathrm{~Hz}$. 2-D and 3-D field calculations minimised multipole components in the C-shaped dipole [8], but a method to fabricate such small laminated $90^{\circ}$ magnets was needed. The key to our design solution was the availability of $1.5 \mathrm{~mm}$ thick modified 1006 sheet steel pre-coated with a B-stage epoxy resin (Remisol EB-540). A 4 to 6 micron thick layer of this resin, which serves as both the lamination insulation and bonding agent, is applied to each side of the sheet steel prior to stamping. The $90^{\circ}$ magnet is fabricated from 5 wedge shaped and 2 end pack modules that are individually stacked, baked and precision machined to the required shape prior to mounting on a base plate assembly. The end pack pole ends are machined with a profile to minimise the magnet integrated sextupole component, which ensures a large dynamic aperture at fields up to $1.83 \mathrm{~T}$. The magnet 

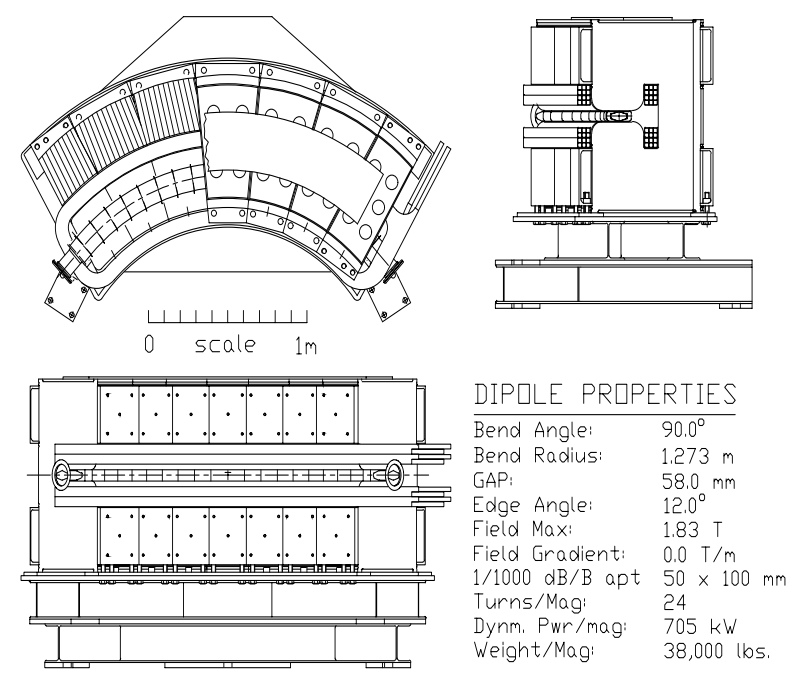

Figure 3: 3 view of a CIS main Dipole Assembly.

assembly is shown in Fig. 3 together with some measured field properties. The $1.5 \mathrm{~mm}$ thick coated steel was also used to fabricate the remainder of the ring laminated magnets ( $2 \mathrm{rf}$ cavity bias quads, 4 trim quads, and 5 ring vertical steering magnets). The steel was sheered to the required perimeter shape, stacked, and baked into blocks in-house and then sent out to be EDM machined to the desired pole tip shape, an economical solution for this small number of laminated magnets. The second challenge for this small ring is beam extraction. The orbit period for $200 \mathrm{MeV}$ protons in CIS is $100 \mathrm{nsec}$. Hence, single turn kick extraction requires a fast kicker with $\leq 50 \mathrm{nsec}(10-90 \%)$ rise time to displace the beam across the $7 \mathrm{~mm}$ thick septum of the Lambertson extraction dipole without loss [6]. A $1.3 \mathrm{~m}$ long parallel plate, $50 \Omega$ Blumlein kicker magnet [10] is installed in straight 2 (Fig. 2). A state-of-the-art pulse forming network and modulator were fabricated by Pulse Sciences, Inc. [11], which reliably delivers \pm 55 $\mathrm{kV}, 300 \mathrm{nsec}$ long pulses to the kicker with a rise time of about $35 \mathrm{nsec} .1 .3 \mathrm{~m}$ is the maximum space available anywhere in CIS, and the $55 \mathrm{kV}$ plate voltage limit is imposed by reliability constraints on the pulse modulator thyratron tube, Application of $\pm 55 \mathrm{kV}$ across the $4 \mathrm{~cm}$ kicker gap is calculated to displace a $200 \mathrm{MeV}$ proton beam $20 \mathrm{~mm}$ at the Lambertson septum entrance. This is enough for efficient extraction if the beam is bumped close to the septum prior to firing the kicker. The dipole trim coils are used to locally bump the beam away from the septum during acceleration and close to the septum for extraction. Kicker EMI during is negligible.

\section{CIS BEAM COMMISSIONING}

A $0.50 \mathrm{~mA}$ (peak) pulsed, $25 \mathrm{keV} \mathrm{H}^{-}$beam from the duoplasmatron is focused at the entrance of the PL-7 linac. The $\mathrm{H}^{-}$beam normalised emittance is $0.6 \pi \mu \mathrm{m}$, smaller than the $1.0 \mu \mathrm{m}$ acceptance of the RFQ. A double einsel lens focuses the beam (125mrad symmetric convergence cone) into the $2.5 \mathrm{~mm}$ diameter RFQ entrance aperture. Beam transmission through the linac is nominally $80 \%$ and the normalised emittance and energy spread of the $7 \mathrm{MeV} \mathrm{H}$ beam are $1.0 \pi \mu \mathrm{m}$ and $150 \mathrm{keV}$ FWHM respectively, in good agreement with specifications.

The $425 \mathrm{MHz}$ linac amplifiers have a $0.2 \%$ duty factor limit. The linac delivers a $200 \mu \mathrm{sec}$ long, $300 \mu \mathrm{A}$ (peak) $7 \mathrm{MeV} \mathrm{H}^{-}$beam pulse at $4 \mathrm{~Hz}$ into a $9 \mathrm{~m}$ transfer beam line matched to the acceptance of CIS. Beam is strip injected on a $6 \mathrm{~mm} \times 25 \mathrm{~mm} 4.5 \mu \mathrm{gm} / \mathrm{cm}^{2}$ carbon foil. Two injection bumpers displace the circulating beam orbit $25 \mathrm{~mm}$ onto the foil for coasting beam accumulation. The ring orbit period at injection is 0.48 $\mu \mathrm{sec}$, hence the maximum theoretical peak intensity gain via strip accumulation is 400 (120 mA). Strip injection efficiency calculations [13] that account for phase space dilution effects of the foil predict an equilibrium gain of $120(36 \mathrm{~mA})$ after $200 \mu \mathrm{sec}$ of accumulation. In practice, an intensity gain of 80 is routinely achieved after $175 \mu \mathrm{sec}$, corresponding to an initial coasting beam accumulation of $8 \times 10^{10}$ protons. Part of the discrepancy between the calculated and measured gain is caused by the emittance growth of the circulating $7 \mathrm{MeV}$ proton beam in the $0.1 \mu$ Torr average ring vacuum. Another source of beam loss at injection is the slow fall time $(0.2$ $\mathrm{msec}$ ) of the bumper magnets after accumulation. Both effects can produce emittance growths nearly equal to that of the foil during injection. Vertical emittance growth during strip accumulation is the intensity limiting mechanism in CIS.

Optimal adiabatic capture of the accumulated beam requires a $2 \mathrm{msec}$ ramp of the RF cavity to $250 \mathrm{~V}$. The captured beam energy is $6.987 \mathrm{MeV}$. The fractional ring tunes are 0.48 horizontal and 0.72 vertical, in excellent agreement with the lattice predictions. The $1 / \mathrm{e}$ lifetime varies from 0.22 seconds to an equilibrium 1.72 seconds in the $200 \mathrm{msec}$ immediately following capture. Beam acceleration is initiated within a few $\mu$ sec of RF capture, by which time lifetime losses reduce the stored beam to $\leq 2 \times 10^{10}$ protons. This intensity is also consistent with the calculated $7 \mathrm{MeV}$ circulating beam space charge limit of $5 \times 10^{10}$ protons, although this has yet to be identified as a CIS intensity limit. Work is continuing to improve the ring vacuum and to reduce the injection bumper fall time to increase stored beam intensity.

\subsection{CIS Acceleration Performance}

Proton beam ramping development began at $50 \mathrm{MeV}$ in November 1997, progressed to $225 \mathrm{MeV}$ by December 1997 , and culminated with the acceleration of $1.1 \times 10^{10}$ protons to $240 \mathrm{MeV}$ in April 1998. The ramp time (seconds), and $\mathrm{n}$ (ramp power) is an integer variable 
from 1 to 5 which is used to vary $\mathrm{dB} \rho / \mathrm{dt}$ from $1.0 \mathrm{Tm} / \mathrm{s}$ $(\mathrm{n}=5)$ to $8 \mathrm{Tm} / \mathrm{s}(\mathrm{n}=1)$ for a $0.5 \mathrm{sec}$ up-ramp to 200 $\mathrm{MeV}$. The software calculates both the main dipole current and RF cavity frequency as a function of $\mathrm{B} \rho$ and creates a 96 vector ramp table for each. Beam acceleration is done open loop (no radial position feedback) via ramping the main dipole current and the accelerator cavity RF frequency. No other ring elements (dipole trims, quads, or steerers) are needed to achieve efficient, centered beam during ramping. 8 x/y BPM pairs and the high bandwidth Wall Gap Monitor (WGM) continuously monitor beam position and intensity around the ring during acceleration. The WGM also measures the circulating beam orbit period and bunching factor. Orbit centering errors are removed manually by making small modifications $(\leq 0.4 \%)$ to the RF cavity frequency ramp vector tables. A software program was recently implemented to read the horizontal BPM positions during acceleration and adjust the $\mathrm{RF}$ frequency vector table automatically. Two iterations of this program are enough to center the beam to $\pm 2 \mathrm{~mm}$, a feature that reduces the setup time for new ramped energies to a matter of minutes.

To date, protons have been accelerated to energies from 50 to $240 \mathrm{MeV}$ with ramp transmissions of $75 \%$ and flattop intensities over $1.1 \times 10^{10}$ protons. All intensity losses occur during the first $200 \mathrm{msec}$ of a 1sec up-ramp and are also caused by gas scattering losses in the $0.1 \mu$ Torr ring vacuum. A beam phase feedback loop added to the RF acceleration system only marginally improves ramp transmission. The horizontal and vertical betatron tunes remain constant during acceleration at $1.485 \pm 0.015$ and $0.72 \pm 0.015$ respectively. The measured bunching factor varies from 3 at injection to about 5 at $225 \mathrm{MeV}$ for RF accelerating voltages above $400 \mathrm{~V}$. The $1 / \mathrm{e}$ lifetime of a $225 \mathrm{MeV}$ proton beam in CIS is $573 \mathrm{sec}$.

The main dipole power supply has sufficient capacity to ramp protons to $250 \mathrm{MeV}$, although the magnets are already driven well into saturation $(1.87 \mathrm{~T})$ at $240 \mathrm{MeV}$. However, insufficient ac line capacity causes significant line "flicker" for ramps above $210 \mathrm{MeV}$ at rep. rates faster than $1 \mathrm{~Hz}$. While higher energy and faster rep. rate development is planned, $200 \mathrm{MeV}$ at $1 \mathrm{~Hz}$ is an ideal beam for Cooler injection. A $0.8 \mathrm{~Hz}, 200 \mathrm{MeV}$ proton ramp cycle consisting of a $0.5 \mathrm{sec}$ up- and downramp, a $50 \mathrm{msec}$ flattop for extraction and a $200 \mathrm{msec}$ reset and fill period was developed for this purpose.

\subsection{CIS Extraction Performance}

The fast kicker and Lambertson septum extraction dipole present the smallest horizontal apertures, $4 \mathrm{~cm}$ and $5 \mathrm{~cm}$ respectively, to the CIS circulating beam. caused no intensity losses at injection when installed, but the Lambertson dipole requires a $7 \mathrm{~mm}$ circulating beam local orbit bump away from the septum to

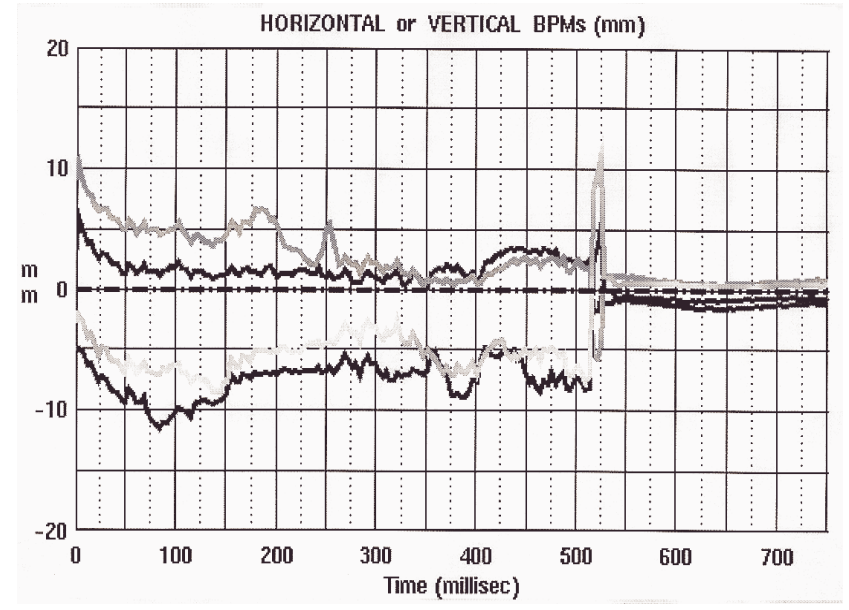

Figure 5: BPM scan for 0.5 ramp to $200 \mathrm{MeV}$ protons.

maintain intensity during acceleration. This local bump is shown in Fig. 5, which is a BPM scan for a $0.5 \mathrm{sec}$ ramp to $200 \mathrm{MeV}$. The kicker magnet is between dipoles 1 and 2, and the Lambertson entrance is just after the exit of dipole 2 (see Fig. 2). The beam positions at the entrance and exit of dipole 1 (top 2 traces) remain nearly centered during the ramp, while the positions in dipole 2 (bottom traces) are moved $-7 \mathrm{~mm}$ to the ring inside to prevent abrupt losses half way up the ramp. The Lambertson vacuum chamber apparently induces these losses, which were not observed prior to its installation. The local bump is inverted at the flattop just prior to extraction, when the beam exiting dipole 2 is bumped $+17 \mathrm{~mm}$ to the septum edge. This can be seen in Fig. 5 beginning at $520 \mathrm{msec}$. Beam is extracted at $525 \mathrm{msec}$.

The Lambertson extraction channel horizontal width is $16 \mathrm{~mm}$. The diameter of a $10 \pi \mu \mathrm{m}, 200 \mathrm{MeV}$ proton beam is about $11 \mathrm{~mm}$, hence a careful alignment of the Lambertson dipole with the extraction orbit is required. Over $1 \times 10^{10}$ protons have been extracted from CIS at 150,200 and $210 \mathrm{MeV}$. Extraction efficiency is typically $75 \%$, and has been as high as $86 \%$. Fig. 6 is a display of the $+55 \mathrm{kV}$ extraction kicker pulse monitor (top), a WGM display of the last 4 orbits of the circulating beam at the flattop (middle), and a BPM

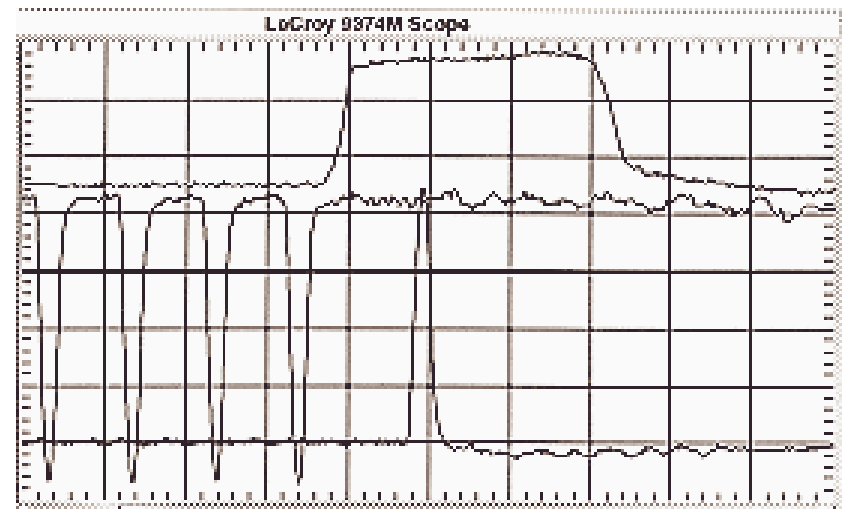

Figure 6: Kicker, WGM and BPM outputs for a 200 $\mathrm{MeV}$ extracted proton beam from CIS. 
signal of the extracted beam in the Cooler transfer beam line (BL9A) for a $200 \mathrm{MeV}$ proton beam. The horizontal scale is $100 \mathrm{nsec} / \mathrm{Div}$. The rise time and width of the 55 $\mathrm{kV}$ kicker pulse are 35 and $300 \mathrm{nsec}$. The orbit period (WGM) of the $200 \mathrm{MeV}$ flattop beam is $100 \mathrm{nsec}$, the FWHM bunch width is $21 \mathrm{nsec}$, and the bunch factor is 4.76. The BPM FWHM signal for the extracted beam is also $21 \mathrm{nsec}$, and measures $7 \times 10^{9}$ protons, $60 \%$ of the $1.1 \times 10^{10}$ protons measured in CIS by the WGM. The extraction kicker for this run was at maximum voltage. In later runs, the kicker plate gap was reduced to $3.7 \mathrm{~cm}$, and the extraction orbit bump was optimised to extract $1.1 \times 10^{10}$ protons of $1.3 \times 10^{10}$ at $200 \mathrm{MeV}(85 \%$ extraction effic.) with a kicker voltage of $\pm 51 \mathrm{kV}$. While careful alignment of the extraction bump is required for this performance, experience has shown that the acceleration and extraction of beam from CIS is quite reproducable.

\section{COOLER INJECTION}

Approximately 9x $10^{9}, 200 \mathrm{MeV}$ protons/pulse at $0.8 \mathrm{~Hz}$ are routinely available from CIS for Cooler injection. A preliminary measurement of the normalised emittance in BL9A is about $18 \pi \mu \mathrm{m}$, and should be considered an upper limit. Transmission through BL9A to the Cooler injection system is $\geqslant 90 \%$. The Cooler injection dipole apertures were recently modified to accept $10 \pi \mu \mathrm{m}$ emittance beams and the Cooler injection ferrite kicker magnet rise time was reduced from $200 \mathrm{~ns}$ to $70 \mathrm{~ns}$ to reduce losses at injection. The single pulse injection efficiency of CIS extracted beam into a Cooler stored orbit is $50 \%$, corresponding to $4 \times 10^{9}$ injected per CIS cycle. Reducing the CIS extracted beam emittance by optimising strip injection parameters may increase Cooler injection efficiency, but has yet to be attempted.

Two methods of CIS beam accumulation in the Cooler have been demonstrated. The first and easiest is coasting beam cooled accumulation where CIS beam is injected and cooled with no Cooler RF cavity voltage. Fig. 7 is a low bandwidth PCT display of this type of accumulation for $200 \mathrm{MeV}$ protons in which beam is accumulated to over $2.4 \mathrm{~mA}$ ( $8 \times 10^{9}$ protons) in $8 \mathrm{sec}$, or 6 pulses from CIS. The vertical scale is $0.5 \mathrm{~mA} / \mathrm{Div}$.

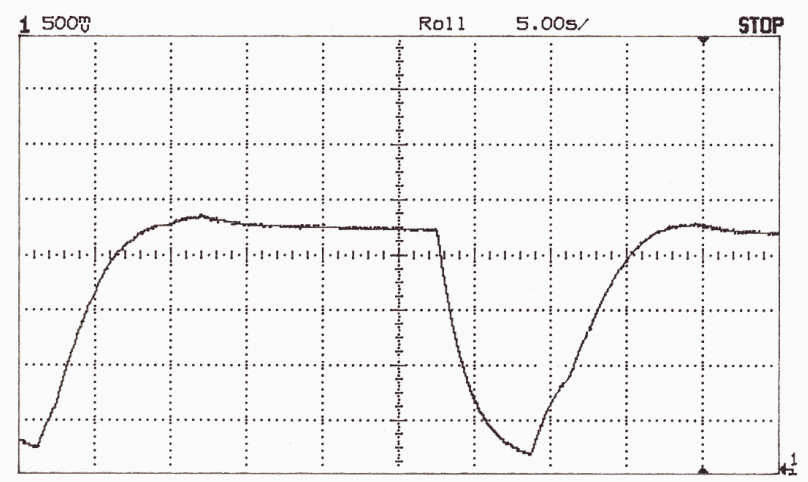

Figure 7: PCT display of $200 \mathrm{MeV}$ proton accumulation
Beam also accumulates to nearly the same current via "sequential" cogging of 5 CIS pulses into the Cooler operating on the $5^{\text {th }}$ harmonic. For this fill method, the timing of each extracted pulse from CIS is synched with the center of each Cooler RF bucket in sequence until all 5 buckets are filled. Beam centering and ramping was demonstrated in the Cooler using the new injection trajectory from CIS. The stored beam energy and 1/e lifetime in the Cooler are $201.57 \mathrm{MeV}$ and $1100 \mathrm{sec}$. The momentum spread of the un-cooled beam injected into the Cooler is $\pm 0.2 \%$, the Cooler design acceptance.

The present record of $3 \mathrm{~mA}$ accumulated in the Cooler with CIS injection is not yet limited by instabilities, but by the availability of development time. CIS/Cooler development competes with the polarised source installation work and Cooler experiment. Cooler experiments began with $200 \mathrm{MeV}$ un-polarised protons from CIS in January 1999 with a significantly increased duty factor and stored beam intensity (1-2 mA average). The stability and reliability of Cooler stored beam is excellent, with intensities varying by less than $10 \%$ from cycle to cycle. Reproducibility is excellent throughout the system and turn-on and setup of the Cooler with CIS injection has been done in as little as 6 hours.

\section{ACKNOWLEDGEMENTS}

Most of the IUCF staff rendered significant technical contributions to the CIS/Cooler project during the last 4 years that made the performance reported here possible. These highly experienced and technically excellent people made this effort with a spirit of optimism and determination that was not justified by the pending future of the facility. It is this spirit and capability that is the essence of IUCF, and which made this an effective research facility for 24 years. My sincere thanks to these, my colleagues.

\section{REFERENCES}

[1] R.E. Pollock, PAC'89, IEEE 89CH2699-0, 17 (1989)

[2] D.L. Friesel et al, PAC'97, IEEE 97CH36167, 2811 (1997)

[3] D.L. Friesel et al, Proc. Linac'98, Chicago, IL, Aug 23-28, 1998.

[4] V. Derenchuk et al, AIP Conf. Proc. 421, 422 (1998).

[5] R.W. Hamm, AccSys Technology, Inc, 11774 Quarry Lane, Pleasanton, CA, 94566

[6] V. Derenchuk et al, PAC'97, IEEE 97CH36167, 2737 (1997).

[7] X. Kang et al, PAC'97, IEEE 97CH36167, 264 (1997).

[8] G.P. Berg et al, PAC'97, IEEE 97CH36167, 3303 (1997).

[9] A. Pei et al, PAC'97, IEEE 97CH36167, 2968 (1997).

[10] M. Ball et al, Proc. $8^{\text {th }}$ Beam Instrumentation Conf, Palo Alto, CA, May 4-7, 1998, to be published.

[11] J. Dinkel et al, PAC'93, IEEE 93CH3279-7, 1357 (1993)

[12] D. Morton, Titan Pulse Sciences, Inc, 600 McCormick St, San Leandro, CA, 94577

[13] K. Hedblom et al, PAC'95, IEEE 95CH35843, 1861 (1995). 